\title{
CONSUMO FARMACOLÓGICO Y SALUD EN PERSONAS MAYORES
}

\author{
Ana Morales Montoya \\ moralesmontoyaana@gmail.com \\ José Manuel Aguilar Parra \\ jmaguilar@ual.es \\ Remedios López Liria \\ rll040@ual.es \\ David Padilla Góngora \\ dpadilla@ual.es \\ Patricia Rocamora Pérez \\ rocamora@ual.es \\ Universidad de Almería
}

Fecha de Recepción: 1 Abril 2018

Fecha de Admisión: 10 Abril 2018

\section{RESUMEN}

El envejecimiento de la población tiene como consecuencia incrementos notables en el numero de enfermedades crónicas y de multimorbilidad, en el consumo de múltiples fármacos y los problemas relacionados con los mismos. El objetivo de este estudio es realizar una revisión sobre el consumo farmacológico en personas mayores de manera que podamos definir los distintos factores que influyen en el consumo de farmacos y el consiguiente gasto sanitario que acarrea. Para ello se ha realizado una revisión sistemática en distintas bases de datos de literatura internacional como Web of Science, Scopus y Pubmed utilizando lo siguientes descriptores: consumo farmacológico mayores, mayores y tratamientos farmacologicos, gasto farmacológico, personas mayores y tratamientos medicos.

Los estudios consultados nos indican que, más del $50 \%$ de todos los medicamentos expendidos son consumidos por adultos mayores; más del $80 \%$ de las personas adultas mayores toman al menos una medicación diaria y $75 \%$ no informa a su médico que usa tratamientos no convencionales. Además, todo lo anterior nos hace ver como mayor parte del incremento del gasto sanitario y social viene determinado por la evolución de distintos factores no demográficos, como son la intensidad de la atención, el coste de los tratamientos o el desarrollo de nuevas tecnologías médicas. Factores, todos ellos, que a diferencia del envejecimiento demográfico, sí son susceptibles de regulación futura y, por tanto, en gran medida socialmente controlables. 


\section{CONSUMO FARMACOLÓGICO Y SALUD EN PERSONAS MAYORES}

El proceso de envejecimiento de la población representa un formidable reto para el sistema sanitario, ya que el gasto sanitario por persona aumenta rápidamente con la edad. Por lo que se hace necesario crear programas y politicas de actuación dirigidos hacia la prevención, los comportamientos y los hábitos saludables.

Palabras clave: consumo farmacológico; mayores; salud; recursos sanitarios

\section{ABSTRACT}

\section{Pharmacological consumption and health for older persons.}

The aging of the population results in notable increases in the number of chronic diseases and multimorbidity, in the consumption of multiple drugs and related problems. The objective of this study is to conduct a review of pharmacological use in older people so that we can define the different factors that influence the consumption of drugs and the consequent health costs involved. To this end, a systematic review was carried out in different databases of international literature such as Web of Science, Scopus and Pubmed using the following descriptors: pharmacological consumption, major and pharmacological treatments, pharmacological expenditure, elderly people and medical treatments.

The studies consulted indicate that more than $50 \%$ of all drugs sold are consumed by older adults; more than $80 \%$ of older adults take at least one medication per day and $75 \%$ do not inform their doctor that they are using unconventional treatments. In addition, all of the above makes us see how most of the increase in health and social spending is determined by the evolution of different non-demographic factors, such as the intensity of care, the cost of treatments or the development of new medical technologies. Factors, all of which, unlike demographic aging, are susceptible to future regulation and, therefore, to a large extent, are socially controllable.

The aging process of the population represents a formidable challenge for the health system, since health spending per person increases rapidly with age. Therefore it is necessary to create programs and policies of action directed towards prevention, behaviors and healthy habits.

Keywords: pharmacological consumption; greater; health; health resources

\section{INTRODUCCIÓN:}

El progresivo envejecimiento poblacional constituye un fenómeno global siendo España uno de los países donde es especialmente evidente. Dado este contexto de cambio demográfico y el hecho de que la población mayor sea el grupo de edad que más recursos sanitarios consume, cobra especial interés la realización de estudios para conocer la frecuencia de la utilización de los servicios sanitarios por las personas mayores y analizar sus factores determinantes. (Cano Pérez et al., 2016)

En España, el proceso de envejecimiento de la población se inició más tarde, pero con una intensidad mayor que en otros países de su entorno. La porción de población mayor de 65 años ha pasado de representar un 11,2\% en 1981 aun 17,3\% en 2011, esperándose que en el año 2050 sea más de un tercio del total de la población española. (Palacios-Ceña et al., 2013). Este dato es de especial relevancia al ser los ancianos el grupo de edad que utiliza con mayor frecuencia los servicios sanitarios.

Las personas mayores presentan un mayor número de visitas médicas, pruebas diagnósticas y prescripciones. Diversos estudios asocian la mayor utilización a factores como edad, peor salud percibida, mayor frecuencia de comorbilidades, nivel educativo bajo o mayor número de tratamientos. La presencia de enfermedades crónicas, principalmente trastornos cardiorrespiratorios, circulatorios y musculo esqueléticos, así como la presencia de malestar psicológico o de trastornos mentales, son factores pueden explicar el elevado consumo farmacológico en los mayores (Somers et al., 2010) (Luciano Devis \& Serrano Blanco, 2008) 
El envejecimiento de la población tiene como consecuencia incrementos en la prevalencia de enfermedades crónicas y de multimorbilidad, en el consumo de múltiples fármacos y los problemas relacionados con los mismos. Se reporta que más del $50 \%$ de todos los medicamentos expendidos son consumidos por adultos mayores; más del $80 \%$ de las personas adultas mayores toman al menos una medicación diaria y $75 \%$ no informa a su médico que usa tratamientos no convencionales. Prescribir de forma apropiada en el adulto mayor es un trabajo difícil que requiere considerar un balance entre los riesgos y beneficios de las medicinas indicadas, las cuales suelen no tener una evidencia clara de su eficacia, dada la poca representatividad de la población adulta mayor en los ensayos clínicos randomizados sobre los cuales se basan las guías clínicas para el manejo de las enfermedades crónicas, condiciones altamente prevalentes en la población adulta mayor. (CasasVásquez, Ortiz-Saavedra, \& Penny-Montenegro, 2016)

La polifarmacia en los adultos mayores, especialmente en aquellos frágiles, está asociada de forma proporcional al deterioro funcional tanto físico como social, a la disminución de la adherencia a fármacos esenciales, a elevados costos tanto para los pacientes como para los propios servicios de salud y al incremento del riesgo de eventos adversos a medicamentos, interacciones medicamentosas, delirio, caídas, hospitalización y muerte (Casas-Vásquez et al., 2016)

Este proceso de envejecimiento demográfico y sobre todo la intensificación a medio plazo del mismo que apuntan todas las proyecciones demográficas, junto con todas las consecuencias anteriormente descritas, ha encendido las señales de alarma sobre la sostenibilidad futura de los actuales niveles de gasto sanitario. La razón que justifica tanta preocupación proviene de un hecho sobradamente conocido: el gasto sanitario de las personas mayores es, en términos relativos, muy superior al del resto de la población. (Casado Marín, 2001)

Constituye el objetivo de este estudio realizar una revisión del estado de la cuestión sobre el notable incremento en el uso de recursos médicos y farmacológicos, por parte de la población mayor en nuestro país, deforma podamos definir cuál es el perfil de ese mayor, así como dotar de estrategias que puedan mejorar este problema social.

\section{DISEÑO Y METODOLOGÍA:}

Hemos realizado una revisión sistemática de documentos de divulgación científica y estudios relacionados con la utilización de los servicios sanitarios por las personas, así como del el consumo farmacológico en este grupo etario.

La búsqueda se llevó a cabo en las siguientes bases de datos de literatura internacional: "Web Of Science", "Scopus" y PubMed.

Las combinaciones de descriptores empleados en español y en inglés han sido las siguientes:

"Consumo farmacológico personas mayores" / "elderly people pharmacological use"

"Gasto farmacológico" / "pharmacological expense"

"Personas mayores + tratamientos médicos" / "elderly people + Medical treatments"

"Personas mayores +servicios sanitarios" / "elderly people + health services"

Para la selección de los trabajos se revisaron en primer lugar el título y el resumen realizando el primer filtro respecto a los objetivos del estudio y posteriormente, tras obtener el texto completo, se seleccionaron solo aquellos que cumplieron los siguientes criterios de inclusión y exclusión:

- Criterios de inclusión: aquellas publicaciones científicas halladas a través de los descriptores indicados, con fecha de publicación posterior a 2013 donde la población objeto de estudio fuera mayor de 60 años.

- Criterios de exclusión: Excluimos aquellos trabajos no relacionados con la utilización de servicios sanitarios en el mayor ni con el consumo farmacológico de estos. 


\section{RESULTADOS Y DISCUSIÓN:}

La utilización de los servicios asistenciales así como de recursos farmacológicos no solo depende de las necesidades de salud de la persona, ya que factores sociodemográficos, psicológicos, médicos y organizativos tienen un papel modulador (Luciano Devis \& Serrano Blanco, 2008).

Tal y como se recoge en el artículo de Aguilar et al., (2015) se ha observado un aumento en la utilización de servicios sanitarios en la población anciana, principalmente en las visitas a Atención Primaria y Atención Especializada. Tanto la mala salud percibida como diagnosticada se asociaron a una mayor frecuentación de servicios sanitarios y de la mayor utilización de fármacos, siendo la salud percibida mejor predictor de la hiperfrecuentación médica y consumo farmacológico que la salud diagnosticada. Esta mayor frecuentación a Atención Especializada, y, por ende, la mayor utilización de fármacos en las clases sociales más altas contrasta con el mejor estado de salud expresado por estas, lo que podría indicar un mayor uso inadecuado de la AE por parte de estos colectivos (Aguilar-Palacio, Carrera-Lasfuentes, Solsona, Sartolo, \& Rabanaque, 2016).

Fernández-Mayoralas et al., (2000), han constatado que la utilización de servicios sanitarios en mayores suele tener como finalidad la obtención de medicación, más que la asistencia sanitaria propiamente dicha. Según datos dela ENS 2011, los principales motivos de la última consulta médica del anciano fueron el diagnóstico de una enfermedad y la revisión, estando en tercer lugar la dispensación de recetas $(23,4 \%)$.

Los problemas de medicación en los adultos mayores siguen siendo frecuentes; se reporta que un tercio de medicamentos son prescritos para personas mayores de 65 años, más del $50 \%$ de todos los medicamentos expendidos son consumidos por adultos mayores, más del $80 \%$ de las personas adultas mayores toman al menos una medicación diaria y $75 \%$ no informa a su médico que usa tratamientos no convencionales, incluidas hierbas medicinales (Casas-Vásquez et al., 2016). España ocupa el segundo lugar en el consumo de fármacos., ocupando el primer Estados Unidos (Sanfélix-Gimeno, Peiró, \& Meneu, 2011)

La gran mayoría de los adultos mayores son portadores de enfermedades crónicas, aquellas situaciones clínicas que tienen duración prolongada, que necesitan atención médica continuada y, a su vez, tienen la capacidad de limitar las actividades de la vida diaria; las prevalencias son más altas en países en desarrollo. La mayoría de los adultos mayores tiene más de una patología en simultaneo, para lo cual, usualmente, reciben múltiples fármacos y, por ende, están expuestos a un mayor riesgo de presentar eventos adversos, interacciones medicamentosas y falta de adherencia

Tal y como recoge el estudio realizado por Casas et al., (2012) La frecuencia de la polifarmacia varía según el ámbito donde se estudie y la definición utilizada. En los Estados Unidos de América, la población adulta mayor, en general, está expuesta a polifarmacia entre 14 a 37\%, siendo mayor al $40 \%$ en residencias geriátricas. En Europa, la frecuencia varía entre 12 al $80 \%$, cifra más alta en residencias geriátricas (23-25). En nuestro país las cifras son similares, cerca del $45 \%$ de los adultos mayores atendidos ambulatoriamente presentan polifarmacia.

Se define polifarmacia al consumo simultáneo de más de tres medicamentos, situación muy común en adultos mayores dada la coexistencia de múltiples comorbilidades. Actualmente, la definición de polifarmacia posee un espectro que va más allá del número y que incluye la pertinencia de la prescripción, es decir, un adulto mayor puede recibir múltiples fármacos si cada uno de ellos posee una eficacia demostrada para las condiciones que presenta, al margen de cuántos sean. Lo contrario ocurre cuando el número de fármacos es elevado gracias a medicinas que son totalmente evitables dada su probada ineficacia, su limitado beneficio para un adulto mayor o su alto riesgo de desarrollar eventos adversos, a esta situación se le ha llamado polifarmacia inadecuada. (Steinman et al., 2006) 
Dada la masiva exposición de la población a los medicamentos, los riesgos asociados a su consumo adquieren una enorme relevancia desde la perspectiva de la salud pública. Probablemente, superior a la de muchas patologías de alta prevalencia.(Sanfélix-Gimeno et al., 2011)

\section{CONCLUSIONES:}

Un problema frecuente que se asocia al incremento en el número de fármacos resulta de la denominada cascada de prescripción, situación que se suscita asumiendo erróneamente que un nuevo síntoma debe ser manejado con un nuevo fármaco y no descartar que se deba a uno de los fármacos recibidos y que, por lo tanto, debiera ser suspendido

Revisar la medicación siempre que se tiene contacto con el paciente en los momentos de evaluación ya sea en consulta ambulatoria u hospitalización, así como detener la administración de medicinas innecesarias son medidas eficaces y seguras que minimizarían la polifarmacia y los eventos adversos

La educación tanto del personal de salud como de los pacientes y sus familiares tiene un efecto positivo en prescribir apropiadamente la medicación. Se deben conocer los cambios relacionados a la edad en la farmacocinética y farmacodinamia, así como las interacciones entre la fragilidad y las medicinas. Los pacientes y familiares deben tomar parte en las decisiones que se toman en relación al tratamiento pues la educación ha demostrado mejorar las tasas de adherencia al tratamiento, así como reducir los reingresos hospitalarios.

Es importante el compromiso de todo el equipo multidisciplinario trabajando en forma coordinada, lo que ha demostrado ser eficaz en mejorar la calidad de la prescripción en el adulto mayor. El equipo multidisciplinario que incluye enfermeras, farmacéuticos y el médico a cargo, deben revisar la lista de medicamentos y estar atentos ante los problemas potenciales derivados del uso continuo de la medicación.

El manejo de las enfermedades crónicas, altamente prevalente en la población adulta mayor y presentes de forma simultánea, se suele basar en recomendaciones

plasmadas como guías clínicas las cuales suelen ser específicas para cada enfermedad individual y extrapoladas de estudios con pacientes usualmente seleccionados sin comorbilidades ni polifarmacia, lo cual dificulta su aplicación en adultos mayores ya que puede elevar el riesgo de deterioro y calidad de vida, si son aplicables de forma rígida sin considerar las particularidades del adulto mayor. Sin embargo, existen directrices y recomendaciones generales que deben de aplicarse de forma integral y apoyados por un equipo multidisciplinario, toda vez que se realice un manejo farmacológico en el adulto mayor. Resulta necesario enfocar más esfuerzos de todas las instituciones educativas de salud en enseñar a los médicos en entrenamiento y a los médicos de atención primaria sobre las particularidades que hacen que el adulto mayor sea más propenso a desarrollar complicaciones derivadas de una mala prescripción.

Desarrollar investigaciones en el área para evaluar el consumo de los fármacos y cuantificar el uso racional de los mismos, posibilitará a las autoridades sanitarias una correcta toma de decisiones en el área del manejo de los recursos terapéuticos farmacológicos disponibles, así como también el análisis de los beneficios, los efectos adversos y el costo económico

La asistencia sanitaria en personas mayores es un tema de comprensión y abordaje complejos. Es necesario revisar las condiciones de nuestro sistema sanitario, para garantizar la equidad en el acceso y evitar que se cumpla la ley de atención inversa, respondiendo así a las mayores necesidades de los colectivos socialmente más desfavorecidos. Aspectos como la salud autopercibida por nuestras personas mayores deberían ser considerados a la hora de predecir la demanda de atención, por encima de la carga de morbilidad. Por último, tenemos un sistema sanitario cuya organi- 


\section{CONSUMO FARMACOLÓGICO Y SALUD EN PERSONAS MAYORES}

zación no atiende a las necesidades de su mayor colectivo de usuarios, con un exceso de especialización y departamentalización, lo que en numerosas ocasiones implica una atención poco adecuada e ineficiente. Resulta ineludible trabajar en una atención centrada en este colectivo, garantizando una adecuada atención sanitaria adaptada al tratamiento y al control de patologías crónicas. Igualmente, es inaplazable el desarrollo de programas cuya finalidad sea una atención integral a las personas en general y a los mayores en particular, en los que colaboren profesionales no sanitarios y en los que se desarrollen medidas sociosanitarias y educativas dirigidas a potenciar el auto-cuidado y la atención de problemas sociales. A menudo, problemas ajenos al sistema de salud se traducen en una demanda de servicios sanitarios por falta de otras alternativas.

\section{REFERENCIAS:}

Antonanzas Villar F, Rodriguez-Ibeas R, Juarez- Castello CA, Lorente Antonanzas MR. Impact of the Royal Decree-Law 16/2012 on the number of prescriptions and pharmaceutical expenditures. Rev Esp Salud Publica 2014 Mar-Apr;88(2):233-249.

Aguilar-Palacio, I., Carrera-Lasfuentes, P., Solsona, S., Sartolo, M. T., \& Rabanaque, M. J. (2016). Utilización de servicios sanitarios en ancianos (España 2006-2012): influencia del nivel de salud y de la clase social. Atencion Primaria, 48(4), 235-243. https://doi.org/ 10.1016/j.aprim.2015.01.016

Cano Pérez, M. D., Castell Alcalá, M. V., Queipo Matas, R., Martín Martín, S., Mateo Pascual, C., \& Otero Puime, Á. (2016). Utilización De Servicios De Atención Primaria, Atención Especializada Y Consumo De Medicamentos Por La Población De 65 Años Y Más En La Comunidad De Madrid. Rev Esp Salud Pública, 90(26), 1-11.

Casado Marín, D. (2001). Los efectos del envejecimiento demográfico sobre el gasto sanitario: mitos y realidades. Gaceta Sanitaria, 15(2), 154-163. https://doi.org/10.1016/S0213-9111(01)71536-1

Casas-Vásquez, P., Ortiz-Saavedra, P., \& Penny-Montenegro, E. (2016). Estrategias para optimizar el manejo farmacológico en el adulto mayor. Revista Peruana de Medicina Experimental Y Salud Pública, 33(2), 335. https://doi.org/10.17843/rpmesp.2016.332.2153

Luciano Devis, J. V., \& Serrano Blanco, A. (2008). Los hiperfrecuentadores en atención primaria: perfil sociodemográfico, características clínicas y propuesta de una nueva definición. Atencion Primaria, 40(12), 631-632. https://doi.org/10.1016/S0212-6567(08)75697-X

Palacios-Ceña, D., Hernández-Barrera, V., Jiménez-García, R., Valle-Martín, B., Fernández-De-LasPeñas, C., \& Carrasco-Garrido, P. (2013). Has the prevalence of health care services use increased over the last decade (2001-2009) in elderly people? A Spanish population-based survey. Maturitas, 76(4), 326-333. https://doi.org/10.1016/j.maturitas.2013.07.016

Sanfélix-Gimeno, G., Peiró, S., \& Meneu, R. (2011). La prescripción farmaceútica en Atención Primaria. Mucho más que un problema de gasto. In La refundación de la Atención Primaria (pp. 53-70).

Somers, A., Robays, H., Vander Stichele, R., Maele, G. V. a N., Bogaert, M., \& Petrovic, M. (2010). Contribution of Drug Related Problems To Hospital Admission. Journal of Nutrition, Health \& Aging, The, 14(17), 477-482.

Steinman, M. A., Seth Landefeld, C., Rosenthal, G. E., Berthenthal, D., Sen, S., \& Kaboli, P. J. (2006). Polypharmacy and prescribing quality in older people. Journal of the American Geriatrics Society, 54(10), 1516-1523. https://doi.org/10.1111/j.1532-5415.2006.00889.x 